\title{
Características del dolor neuropático crónico y su relación con el bienestar psicológico en pacientes con lesión medular
}

\author{
M.D. Soler a, J. Saurí-Ruiz a , M.L. Curcoll-Gallemí a , J. Benito-Penalva ${ }^{\text {a }}$, \\ E. Opisso-Salleras ${ }^{\text {a }}$, A. Chamarro-Lusar ${ }^{\text {b }}$, J. Vidal-Samsó ${ }^{a}$
}

\begin{abstract}
CARACTERÍSTICAS DEL DOLOR NEUROPÁTICO CRÓNICO Y SU RELACIÓN
CON EL BIENESTAR PSICOLÓGICO EN PACIENTES CON LESIÓN MEDULAR

Resumen. Objetivos. Determinar la prevalencia del dolor neuropático $(D N)$ en una muestra de pacientes con lesión medular (LM), examinar la relación entre las características clínicas, demográficas y psicológicas con la prevalencia del DN después de una LM y a su vez con los diferentes tipos de DN, y estudiar el valor predictivo de los factores más significativos. Pacientes y métodos. Estudio transversal observacional de un total de 260 pacientes que acudieron a una revisión anual. Las principales medidas de resultados incluyeron presencia/ausencia de DN, índice de bienestar psicológico, historia detallada del dolor y escala de puntuación numérica de 0-10 para la media de intensidad del dolor. Resultados. 93 pacientes (36\%) con LM tenían DN. De los pacientes que presentaron DN, un 35\% mostró dolor a nivel de la lesión, mientras que un $65 \%$ presentó DN por debajo. La única variable con capacidad predictiva sobre la prevalencia del DN fue la edad en el momento de la LM. Se observaron diferencias estadísticamente significativas entre la prevalencia del DN y la percepción de bienestar psicológico; el grupo de pacientes con DN fue el que tuvo proporcionalmente un malestar psicológico mayor. En cuanto a los diferentes tipos de DN, las variables con capacidad predictiva sobre la clasificación del DN eran el inicio temprano del dolor en el DN a nivel y el hecho de que la lesión sea incompleta en el DN por debajo. Conclusión. El DN aparece con frecuencia después de la LM y se da de forma más común en aquellos pacientes que han sufrido la lesión a edades más avanzadas. El DN tiene un impacto negativo en la percepción de bienestar psicológico. [REV NEUROL 2007; 44: 3-9]

Palabras clave. Bienestar psicológico. Dolor a nivel. Dolor neuropático crónico. Dolor por debajo. Lesión medular. Prevalencia.
\end{abstract}

\section{INTRODUCCIÓN}

El dolor crónico en la lesión medular se ha reconocido como una consecuencia significativa y frecuente tras la lesión medular (LM) [1-6]. El dolor crónico se ha mencionado a menudo como uno de los factores más importantes de la pérdida de calidad de vida y bienestar psicológico tras una discapacidad física [3,6-8], y condiciona significativamente el afrontamiento y la adaptación a la lesión medular [9,10].

En el año 2000, la Asociación Internacional para el Estudio del Dolor -Association for the Study of Pain (IASP)- publicó una nueva clasificación del dolor [11] que aglutina todos los tipos de dolor tras una LM. Categoriza el dolor en términos de su localización relativa al nivel de la lesión y distingue el dolor neuropático (DN) del dolor nociceptivo. La distinción del DN en relación con el nivel de la lesión (dolor a nivel, dolor por debajo de la lesión) se sustenta en posibles mecanismos subyacentes a este tipo de dolor; se incluyen mecanismos tanto periféricos como centrales. Mientras que el dolor por debajo de la lesión se considera como dolor central causado por el daño en la médula espinal, el dolor a nivel de la lesión tiene componentes

\footnotetext{
Aceptado tras revisión externa: 12.09.06.

${ }^{a}$ Hospital de Neurorrehabilitación. Instituto Guttmann. Universitat Autònoma de Barcelona. Badalona, Barcelona. ${ }^{b}$ Facultad de Psicología, Ciencias de la Educación y del Deporte Blanquerna. Universitat Ramon Llull. Barcelona, España.

Correspondencia: Dra. M. Dolores Soler. Hospital de Neurorrehabilitación. Instituto Guttmann. Unidad de Lesionados Medulares. Camí de Can Ruti, s/n. E-08916 Badalona (Barcelona).E-mail: dsoler@guttmann.com

Agradecimientos. A Teresa Marqués, por su colaboración en la obtención de la información; a Touria Louah, por su apoyo en la búsqueda bibliográfica, y al Dr. Xavier Navarro, de la Universitat Autònoma de Barcelona, por su apoyo continuo, su valiosa aportación y sus consejos.

(C) 2007, REVISTA DE NEUROLOGÍA
}

centrales y periféricos que son difíciles de separar [12]. El DN se correlaciona con la cantidad de sustancia gris dañada por encima y por debajo del sitio principal de la lesión [13] y con la cantidad de daño celular secundario causado por la liberación de neurotransmisores (glutamato y N-metil-D-aspartato) [14] y citocinas inflamatorias $[15,16]$. El DN por debajo está asociado con problemas axonales, con la pérdida o el daño a lo largo del tracto espinotalámico [17].

Pocos estudios determinan específicamente la prevalencia del DN en la población de lesionados medulares y la variabilidad de los datos refleja la falta de un sistema clasificatorio consensuado. Este tipo de dolor se caracteriza por su persistencia temporal y por su heterogeneidad; como hemos mencionado, no es posible atribuirlo a un solo factor etiopatogénico o a una sola lesión anatómica; son varios los mecanismos posiblemente implicados [18]. Los métodos terapéuticos empleados, en su mayor parte empíricos, resultan a menudo poco efectivos porque los mecanismos patogénicos implicados en este tipo de dolor, en gran parte, todavía se desconocen $[12,19]$.

Se han asociado numerosos factores físicos con el desarrollo y la cronicidad del dolor después de una lesión medular, y se han obtenido resultados dispares. En relación con el DN, en los últimos años se han analizado factores como el nivel de la lesión [3,15], la lesión completa o incompleta [3,20-24], el tipo de lesión [24,25] y el género [2,24,25]; los aspectos relacionados con la edad son los que han mostrado resultados más consistentes [21,23-26].

Con relación al dolor y los aspectos emocionales, la correlación entre el malestar emocional (por ejemplo, depresión, estrés, ansiedad, fatiga), la menor calidad de vida y el dolor crónico se ha demostrado consistentemente en diversos trabajos [3, 23]. Störmer et al [2] encontraron que el dolor crónico, las disestesias y las variables psicológicas estaban más estrechamen- 
te relacionadas que el dolor y las variables médicas. Otros autores [6,27] han constatado la existencia de una percepción mayor del estrés, una aceptación baja de la lesión y una valoración pobre de la salud con relación al dolor crónico en pacientes con LM.

Los objetivos del presente estudio han sido:

- Determinar la prevalencia del DN en pacientes afectados por una LM.

- Examinar la relación entre las características clínicas, demográficas y psicológicas con la prevalencia del DN después de una LM y a su vez con los diferentes tipos de DN.

- Estudiar los factores con un valor predictivo mayor en el desarrollo del DN.

\section{PACIENTES Y MÉTODOS}

Se realizó un estudio transversal observacional. La muestra estaba formada por 272 pacientes que acudieron a la revisión periódica anual en la consulta externa de la unidad de lesionados medulares del Hospital de Neurorrehabilitación Institut Guttmann de Badalona (Barcelona), de noviembre de 2003 a junio de 2004. Se consideraron sujetos de estudio todos aquellos pacientes mayores de 16 años, diagnosticados de una lesión medular y con el tratamiento rehabilitador finalizado. Del total de la muestra se excluyó del estudio a 12 pacientes que no cumplían los criterios mencionados.

\section{Datos sociodemográficos y características de la lesión}

Se obtuvieron datos de la historia clínica de cada paciente relacionados con su lesión: edad actual, sexo, edad al contraer la lesión y etiología. Los pacientes se dividieron en cinco grupos en función de su edad en el momento de la lesión: 0-19 años $(n=43), 20-29$ años $(n=56), 30-39$ años $(n=43), 40-49$ años $(n=55)$ y más de 50 años $(n=63)$. Se definieron como etiología traumática todas aquellas lesiones causadas por diferentes tipos de traumatismo (accidentes de tráfico, caídas fortuitas, accidentes laborales o deportivos, etc.) y como etiología médica aquellas lesiones medulares causadas por enfermedades como tumores, mielitis víricas o infecciosas, isquemias medulares, etc. El nivel neurológico de la lesión y el grado de discapacidad se valoraron según los estándares internacionales para la clasificación neurológica y funcional de la lesión medular ASIA (American Spinal Injury Association) [28,29]. Al realizar el análisis estadístico, la variable ASIA se dicotomizó en dos categorías: ASIA A (lesión completa) y ASIA B, C y D (lesión incompleta). El nivel lesional se clasificó en dos categorías (paraplejía y tetraplejía). El tipo de lesión tras la lesión medular se determinó basándose en los hallazgos clínicos neurológicos. Las lesiones de cono medular y cauda equina se definieron como lesiones espinales en la región toracolumbar en las que estaba implicada la médula (cono medular) o en las que el daño parece confinado a las raíces nerviosas en el caso de la cauda equina.

\section{Dolor neuropático}

Un médico rehabilitador diagnosticó el DN siguiendo los criterios diagnósticos propuestos por la IASP [11] y atendiendo a la localización y la descripción del dolor. De acuerdo con la clasificación de la IASP, partimos de dos categorías de DN tras una lesión medular: el DN a nivel se definió como ardiente, punzante, eléctrico y localizado en los dermatomas del nivel de la lesión o justo por encima de ésta. El dolor con estos descriptores en las piernas en los pacientes con el nivel de la lesión en la cauda equina se clasificó como DN a nivel. El DN por debajo se definió como ardiente, punzante, como un disparo, localizado de forma difusa por debajo del nivel de la lesión. Los pacientes que no presentaban síntomas de DN formaron parte del grupo control. No se tuvieron en cuenta en este estudio otros tipos de DN incluidos en las categorías de la IASP [11] como son los DN por encima de la lesión, el síndrome de dolor regional complejo o las mononeuropatías compresivas.

\section{Descriptores del dolor}

A todos los participantes se les administró una lista con 24 adjetivos y se les pidió que señalaran aquellos que describían mejor su experiencia actual de dolor $[30,31]$.

\section{Intensidad del dolor}

La intensidad del dolor se evaluó utilizando la escala de puntuación numérica [32] en la cual el sujeto puntúa con un cero cuando no siente dolor y con un 10 el dolor más intenso imaginable; dispone del resto de la escala para valorar su grado de dolor. Este tipo de escalas ha mostrado su validez y fiabilidad; tiene la ventaja de que se puede administrar oralmente y esto la hace más fácil de utilizar en personas con dificultades motrices.

Los valores numéricos de la intensidad se agruparon en tres categorías: se consideraron dolor leve los valores menores o iguales a 3; moderado, entre 4 y 7 , e intenso, los valores mayores de 7 .

\section{Percepción de bienestar psicológico}

Estábamos interesados en obtener datos sobre la percepción subjetiva de bienestar psicológico de nuestros pacientes porque se ha comprobado que las sensaciones subjetivas se relacionan más estrechamente con el bienestar psicológico y físico que con otras variables de carácter más objetivo. Por este motivo, se utilizó el índice de bienestar psicológico (IBP) de Dupuy [33], adaptado al español por Badía et al [34]. El bienestar psicológico se refiere a los sentimientos subjetivos de bienestar o malestar en los adultos. Explica cómo se sienten los individuos sobre su propio estado de salud.

El IBP incluye 22 ítems agrupados en seis dimensiones: ansiedad, depresión, autocontrol, sentimiento de bienestar, vitalidad y salud general. Cada ítem puede responderse en una escala Likert con seis categorías de respuesta que representan diferentes grados de intensidad o frecuencia del ítem en la última semana. La puntuación total puede ir de 22 (malestar grave) hasta 132 (bienestar positivo), y también pueden obtenerse puntuaciones para cada dimensión. Los intervalos de la puntuación global son: malestar grave (22-82), malestar moderado (83-94) y bienestar positivo (95-132).

Las puntuaciones del cuestionario se dividieron en dos categorías: bienestar psicológico (95-132) y malestar psicológico (22-94).

\section{Análisis de datos}

Se realizó un primer nivel de análisis para valorar la prevalencia del DN en el conjunto de la muestra. Las comparaciones entre el grupo de pacientes con DN y el grupo control se realizaron mediante los estadísticos $\chi^{2}$ y $V$ de Cramer.

Para valorar las diferencias de edad, la edad en el momento de la lesión, el tiempo desde la lesión y la percepción de bienestar psicológico en función de la prevalencia del DN se calcularon sucesivos ANOVA de un factor. Se consideró significativo un valor $p<0,05$.

Para pronosticar la prevalencia de $\mathrm{DN}$ a partir de los factores de riesgo (edad, género, nivel de la lesión, ASIA, etiología y percepción de bienestar psicológico) se utilizó la regresión logística binaria.

Se realizó un segundo grupo de análisis en el que se incluyó únicamente a los pacientes con DN a nivel de la lesión y por debajo de ésta. Para ello se descartó a 22 pacientes que registraron tener los dos tipos de dolor a la vez, o a aquellos que en su descripción de los síntomas mostraban elementos de confusión, con el objetivo de incidir en aquellos factores que pueden intervenir específicamente en cada uno de los tipos de dolor. En estos análisis se hicieron las comparaciones entre ambos grupos mediante el uso de los estadísticos $\chi^{2}$ y $V$ de Cramer. Se calcularon sucesivos ANOVA para valorar las diferencias en edad, la edad en el momento de la lesión, el tiempo desde la lesión y el tiempo de evolución del dolor en función de la clasificación del dolor (a nivel/por debajo). La regresión logística se utilizó para pronosticar la clasificación del dolor basándose en los factores de riesgo posibles (nivel de la lesión, ASIA, etiología, inicio del dolor). El análisis de los datos se realizó con el paquete estadístico SPSS 12.0.

\section{RESULTADOS \\ Datos demográficos}

La media de edad del total de la muestra fue de 50,5 años (desviación estándar, $\mathrm{DE}=16,7) .187$ pacientes $(71,9 \%)$ eran hombres y $73(28,1 \%)$ eran mujeres. Los datos demográficos se resumen en la tabla I.

\section{Prevalencia del DN después de la LM}

De los 260 pacientes que formaban el total de la muestra, 93 (35,8\%) padecían DN. De los pacientes que presentaron DN, 25 (35,2\%) mostraron dolor a nivel y $46(64,8 \%)$ presentaron DN por debajo de acuerdo con los criterios de clasificación de la IASP, mientras que 22 pacientes presentaron ambos 


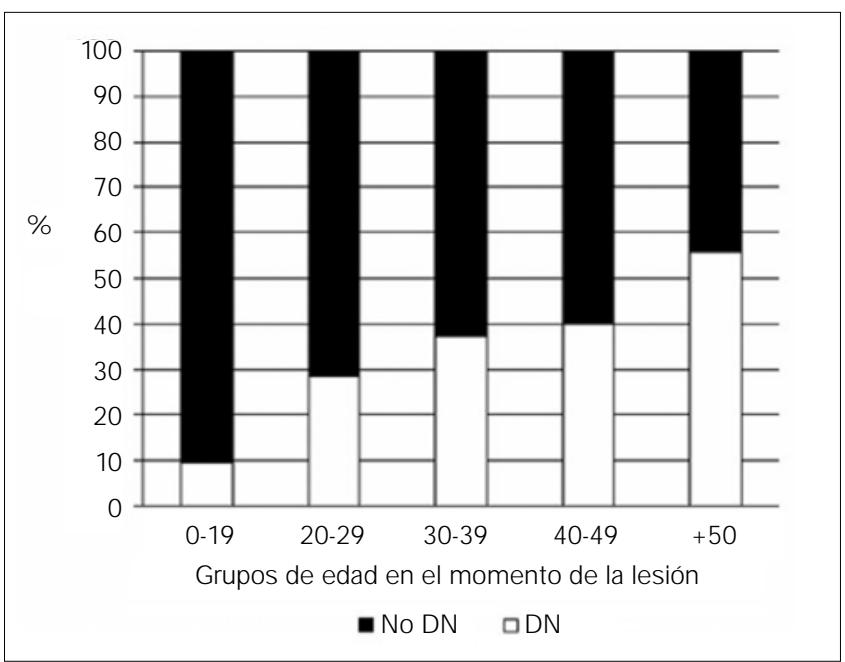

Figura 1. Prevalencia del dolor neuropático en los diferentes grupos de edad en el momento de la lesión.

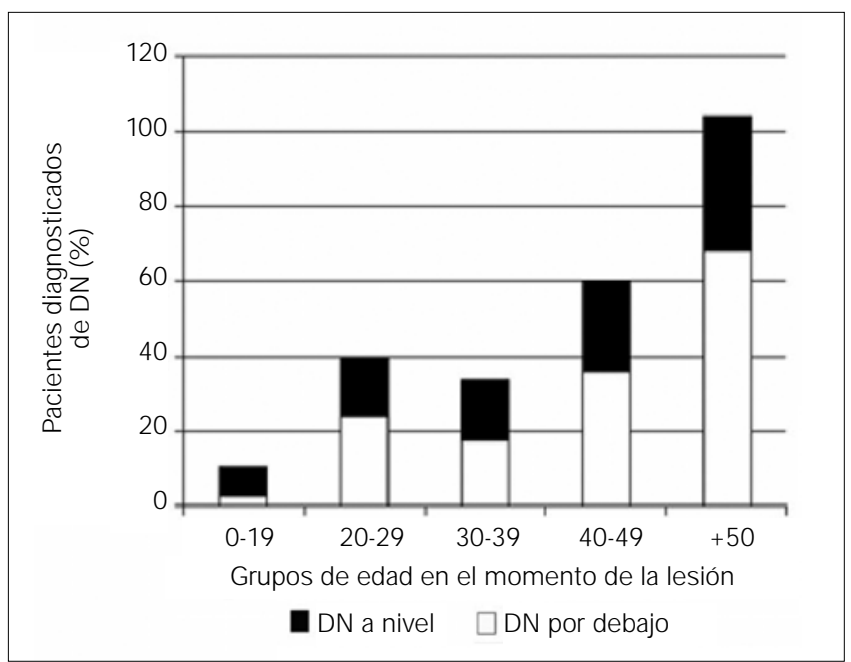

Figura 2. Tipos de dolor neuropático en los diferentes grupos de edad.
Tabla I. Datos demográficos y características de la lesión.

\begin{tabular}{|c|c|c|c|c|}
\hline & $\begin{array}{l}\text { Muestra total } \\
(n=260)\end{array}$ & $\begin{array}{l}\text { DN } \\
(n=93)\end{array}$ & $\begin{array}{l}\text { No DN } \\
(n=167)\end{array}$ & $p$ \\
\hline & Media (DE) & Media (DE) & Media (DE) & \\
\hline Edad (años) & $50,5(16,7)$ & $54,4(15,9)$ & $48,3(16,9)$ & 0,005 \\
\hline $\begin{array}{l}\text { Edad en el momento } \\
\text { de la lesión (años) }\end{array}$ & $37,5(18,1)$ & $44,1(16,08)$ & $33,9(18,1)$ & 0,000 \\
\hline $\begin{array}{l}\text { Tiempo de evolución } \\
\text { (años) }\end{array}$ & $12,9(9,4)$ & $10,2(8,9)$ & $14,4(9,3)$ & 0,001 \\
\hline \multicolumn{5}{|l|}{ Sexo } \\
\hline Masculino & $187(71,9 \%)$ & $66(71 \%)$ & $121(72,5 \%)$ & \multirow{2}{*}{0,79} \\
\hline Femenino & $73(28,1 \%)$ & $27(29 \%)$ & $46(27,5 \%)$ & \\
\hline \multicolumn{5}{|c|}{$\begin{array}{l}\text { Edad de los grupos (en } \\
\text { el momento de la lesión) }\end{array}$} \\
\hline 0-19 años & $43(16,5 \%)$ & $4(4,3 \%)$ & $39(23,4 \%)$ & \multirow{5}{*}{0,000} \\
\hline 20-29 años & $56(21,5 \%)$ & $16(17,2 \%)$ & $40(24,0 \%)$ & \\
\hline 30-39 años & $43(16,5 \%)$ & $16(17,2 \%)$ & $27(16,2 \%)$ & \\
\hline 40-49 años & $55(21,2 \%)$ & $22(23,7 \%)$ & $33(19,8 \%)$ & \\
\hline$>50$ años & $63(24,2 \%)$ & $35(37,6 \%)$ & $28(16,8 \%)$ & \\
\hline
\end{tabular}

Gravedad de la lesión (ASIA)

\begin{tabular}{lllll}
\hline A & $142(54,6 \%)$ & $50(53,8 \%)$ & $92(55,1 \%)$ & \multirow{2}{*}{0,83} \\
\cline { 1 - 2 } B-D & $118(45,4 \%)$ & $43(46,2 \%)$ & $75(44,9 \%)$ & \\
\hline
\end{tabular}

Etiología

\begin{tabular}{lrrrr}
\hline Traumática & $164(63,1 \%)$ & $52(55,9 \%)$ & $112(67,1 \%)$ & \multirow{2}{*}{0,07} \\
\cline { 1 - 3 } Médica & $96(36,9 \%)$ & $41(44,1 \%)$ & $55(32,9 \%)$ & \\
\hline
\end{tabular}

Nivel de la lesión

\begin{tabular}{lrrrr}
\hline Paraplejía & $183(70,4 \%)$ & $70(75,3 \%)$ & $113(67,7 \%)$ \\
\cline { 1 - 3 } Tetraplejía & $77(29,6 \%)$ & $23(24,7 \%)$ & $54(32,3 \%)$ &
\end{tabular}

DN: dolor neuropático; DE: desviación estándar; ASIA: American Spinal Injury Association. tipos de DN y se los excluyó del análisis estadístico. Respecto a la muestra general, el porcentaje de sujetos con DN a nivel es del $13,8 \%$, y el de sujetos con DN por debajo, del $21,95 \%$.

\section{Prevalencia del DN con relación a la edad}

La prevalencia del DN se incrementa de forma estadísticamente significativa con la edad $(F=8,04 ; d f=1 ; p=$ $0,005)$ y la edad en el momento de la lesión $(F=20,26$; $d f=1 ; p=0,000)$. El grupo de edad mayor de 50 años en el momento de la instauración de la LM presentó la prevalencia más alta $(55,6 \%)$ y mostró diferencias estadísticamente significativas con respeto a los demás grupos de edad $\left(\chi^{2}=25,57 ; d f=4 ; p=0,000\right)$ (Fig. 1). El tiempo de evolución trascurrido desde la LM fue significativamente mayor para el grupo control respeto del grupo de pacientes con DN $(F=12,11 ; d f=1 ; p=0,001)$. En la figura 2 se puede observar la prevalencia de los diferentes tipos de DN en cada grupo de edad en el momento de la lesión. En todos los grupos el porcentaje de pacientes con DN por debajo es mayor excepto en el grupo de edad 0-19. Sin embargo, no se observaron diferencias estadísticamente significativas $\left(\chi^{2}=0,26 ; d f=3\right.$; $p=0,96)$.

\section{Otros datos relacionados con la edad}

Las lesiones completas eran más frecuentes en los sujetos más jóvenes en el momento de la LM, de manera que, a medida que aumentaba la edad, las lesiones incompletas eran más frecuentes $\left(\chi^{2}=16,47 ; d f=4, p=0,002\right)$. No se encontraron diferencias significativas entre los grupos de edad para el tipo de lesión (paraplejía/tetraplejía) $\left(\chi^{2}=1,65 ; d f=1 ; p=0,19\right)$. También se observó que en los grupos de edad más jóvenes había una tendencia a que la etiología traumática fuese más común; a medida que la edad aumentaba la tendencia se invertía y en los grupos de mayor edad la etiología médica era la predominante $\left(\chi^{2}=69,79 ; d f=4 ; p=0,000\right)$.

En cuanto a la percepción de bienestar psicológico, aunque las puntuaciones totales del cuestionario IBP tendían a ser inferiores a medida que aumentaba la edad, no se registraron diferencias significativas $(F=2,03 ; p=0,09)$. Tampoco se registraron diferencias significativas en cuanto al malestar o bienestar psicológico entre los grupos de edad en el momento de la lesión $\left(\chi^{2}=5,89 ; d f=4 ; p=\right.$ 0,$20 ; V$ de Cramer $=0,17$ ). 


\section{Relación entre el DN, el nivel de la lesión y la etiología}

Con relación a la muestra total, la presencia de DN no se relaciona con el nivel neurológico de la lesión $\left(\chi^{2}=1,65 ; d f=1 ; p=0,19\right)$. Sin embargo, cuando se analizan los diferentes tipos de DN se observan diferencias estadísticamente significativas $\left(\chi^{2}=4,67 ; d f=1 ; p=0,03\right)$. Así, el 41,8\% de los parapléjicos y el $12,5 \%$ de los tetrapléjicos mostraron dolor a nivel, mientras que el $58,2 \%$ de los parapléjicos y el $87,5 \%$ de los tetrapléjicos mostraron dolor por debajo. Con relación al total, la presencia de DN no se relaciona con la etiología de la lesión $\left(\chi^{2}=3,19 ; d f=1 ; p=0,07\right)$. Al analizar los diferentes tipos de DN tampoco se encontraron diferencias estadísticamente significativas $\left(\chi^{2}=1,28 ; d f=1 ; p=0,257\right)$.

\section{Relación entre DN y gravedad de la lesión según la escala ASIA}

La presencia de DN no se relacionó con la gravedad de la lesión según la escala ASIA $\left(\chi^{2}=0,04 ; d f=1 ; p=0,83\right)$. Sin embargo, cuando se analizaron los diferentes tipos de DN se registraron diferencias estadísticamente significativas $\left(\chi^{2}=6,06 ; d f=1 ; p=0,01\right)$. De aquellos que tenían una lesión completa, el $26,8 \%$ mostró dolor a nivel y el $29,6 \%$ dolor por debajo, mientras que de aquellos pacientes con una lesión incompleta, el 8,5\% mostró dolor a nivel de la lesión y el $35,2 \%$ dolor por debajo.

\section{Inicio del DN}

El inicio del dolor tendía a ser más temprano en pacientes con DN a nivel que en los pacientes con DN por debajo $\left(\chi^{2}=7,15 ; d f=2 ; p=0,02\right)$. Los síntomas de DN empezaron durante los tres primeros meses después de la lesión en el $80 \%$ de los pacientes con dolor a nivel y en el $47,8 \%$ de los pacientes con dolor por debajo. El DN empezó entre tres meses y un año después de la LM en el $16 \%$ de los pacientes con dolor a nivel y en el $34,8 \%$ de los pacientes con dolor por debajo, mientras que el $4 \%$ de los pacientes con dolor a nivel y el $17,4 \%$ de los pacientes con dolor por debajo manifestaron que su dolor empezó más de un año después de la LM (Fig. 3).

\section{Intensidad del dolor y descriptores}

La media total de la intensidad del DN fue de 6,96 (DE = 1,81). Los pacientes con DN a nivel tenían una intensidad ligeramente superior del dolor $(7,12$; $\mathrm{DE}=1,98)$ que los pacientes con $\mathrm{DN}$ por debajo $(6,87 ; \mathrm{DE}=1,73)$. Del total, 30 pacientes $(42,3 \%)$ calificaron la intensidad de su dolor como moderado mientras que 41 pacientes $(57,7 \%)$ lo calificaron como grave. En este análisis no se registraron diferencias estadísticamente significativas entre las dos categorías de dolor $\left(\chi^{2}=0,80 ; d f=1 ; p=0,77\right)$. Tampoco se hallaron diferencias estadísticamente significativas entre la gravedad del dolor y las variables médicas. En los pacientes con DN, los descriptores del dolor más frecuentes fueron 'ardiente' $(47,9 \%)$, 'hormigueo' $(42,3 \%)$ y 'persistente' $(40,8 \%)$ (Tabla II).

\section{Percepción de bienestar psicológico}

En los pacientes que mostraron DN, la media del cuestionario IBP fue de $98,18(\mathrm{DE}=15,01)$ y de $106,2(\mathrm{DE}=14,40)$ en los pacientes que no mostraron este tipo de dolor $(F=14,78 ; p=0,000)$ (Fig. 3). Estas diferencias se mostraron en las subescalas de salud general, bienestar, depresión y vitalidad del cuestionario pero no en las subescalas de ansiedad y autocontrol (Tabla III). El grupo de pacientes con DN fue el que tenía proporcionalmente más malestar psicológico $\left(\chi^{2}=4,38 ; d f=1 ; p=0,036\right)$. En cuanto a los diferentes tipos de DN, no se observaron diferencias estadísticamente significativas con relación a la percepción de bienestar psicológico $(F=0,178 ; p=0,178)$.

\section{Predictores de la prevalencia y de la clasificación del DN}

Los resultados del análisis de regresión logística sobre la prevalencia del DN a partir de los factores físicos (nivel neurológico, ASIA, etiología) y demográficos (grupos de edad en el momento de la lesión) mostraron que la única variable con capacidad predictora sobre la prevalencia del DN fue la edad en el momento de la LM (Wald $=21,53 ; d f=1 ; p=0,000)$. Los resultados indican que la edad en el momento de la lesión predice correctamente al $66,5 \%$ la clasificación de la muestra $(82,9 \%$ de los sujetos sin dolor y $37,6 \%$ de los sujetos con dolor). El índice de especificidad era de 0,83 , y el de sensibilidad, de 0,55. El coeficiente de determinación muestra que esta variable explica únicamente el $12 \%$ de la varianza de la prevalencia del DN, por lo cual se puede considerar como un predictor pobre.

Los resultados del análisis de regresión logística sobre la clasificación del DN (a nivel, por debajo) a partir de las variables médicas (nivel neuroló-

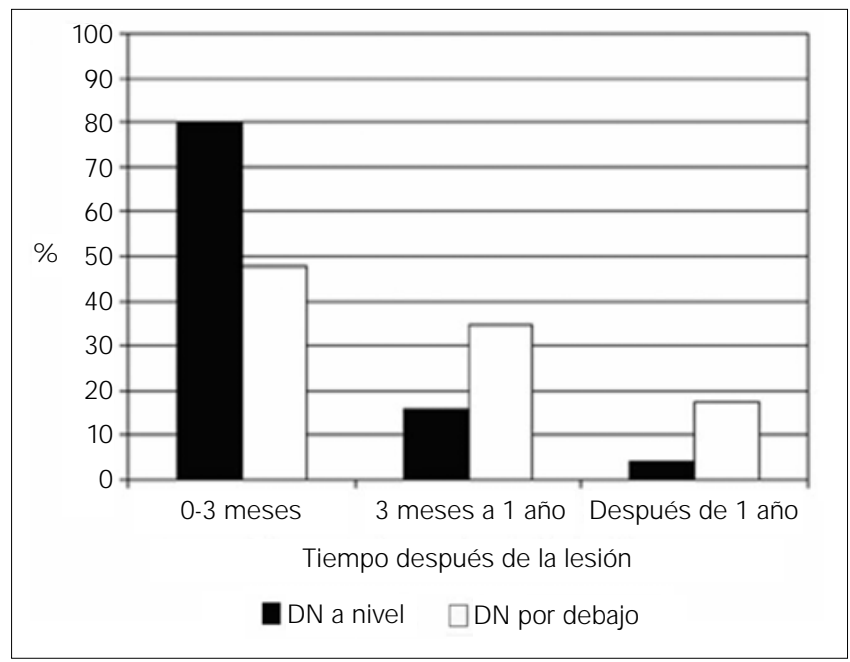

Figura 3. Inicio del dolor neuropático después de la lesión medular.

Tabla II. Descriptores del dolor indicados por los pacientes con lesión medular según el tipo de dolor.

\begin{tabular}{llcl}
\hline & $\begin{array}{l}\text { Muestra total DN } \\
(n=71)\end{array}$ & $\begin{array}{l}\text { DN a nivel } \\
(n=25)\end{array}$ & $\begin{array}{l}\text { DN por debajo } \\
(n=46)\end{array}$ \\
\hline Ardiente & $34(47,9 \%)$ & $8(32 \%)$ & $26(56,5 \%)$ \\
\hline Hormigueo & $30(42,3 \%)$ & $7(28 \%)$ & $23(50 \%)$ \\
\hline Persistente & $29(40,8 \%)$ & $7(28 \%)$ & $22(47,8 \%)$ \\
\hline Aprieta & $26(36,6 \%)$ & $9(36 \%)$ & $17(37 \%)$ \\
\hline Punzante & $24(33,8 \%)$ & $13(52 \%)$ & $11(23,9 \%)$ \\
\hline Eléctrico & $23(32,4 \%)$ & $8(32 \%)$ & $15(32,6 \%)$ \\
\hline Calambre & $18(25,4 \%)$ & $6(24 \%)$ & $16(34,8 \%)$ \\
\hline Comiente & $20(28,2 \%)$ & $9(36 \%)$ & $11(23,9 \%)$ \\
\hline Agudo & $18(25,4 \%)$ & $10(40 \%)$ & $8(17,4 \%)$ \\
\hline Palpitante & $2(2,8 \%)$ & $2(8 \%)$ & $0(0 \%)$ \\
\hline
\end{tabular}

DN: dolor neuropático.

gico, ASIA, etiología, inicio del dolor) mostraron que el ASIA (Wald $=$ $4,84 ; d f=1 ; p=0,02)$ y el inicio del dolor $($ Wald $=5,26 ; d f=1 ; p=0,02)$ son las únicas variables que tienen capacidad predictiva sobre la clasificación del DN. Las dos variables clasifican correctamente al 74,6\% de los sujetos $(78,3 \%$ de los sujetos con DN por debajo del nivel de la lesión y $68 \%$ de los sujetos con DN a nivel de la lesión). El índice de sensibilidad era de 0,68 , y el de especificidad, de 0,78. El coeficiente de determinación muestra que las dos variables predictoras explican conjuntamente el $22,6 \%$ de la varianza de la clasificación del DN.

\section{DISCUSIÓN}

Los resultados del presente estudio aportan datos relevantes respecto a las características del DN en personas con LM. De los 260 pacientes que integraban la muestra del estudio, un $36 \%$ presentaba DN. De los pacientes con DN, un 35\% tenía dolor al nivel de la lesión, mientras que un $65 \%$ tenía dolor por debajo de la lesión. La única variable con valor predictivo de la presencia de DN fue la edad en el momento de la instauración de la LM. También hallamos una relación significativa entre la pre- 


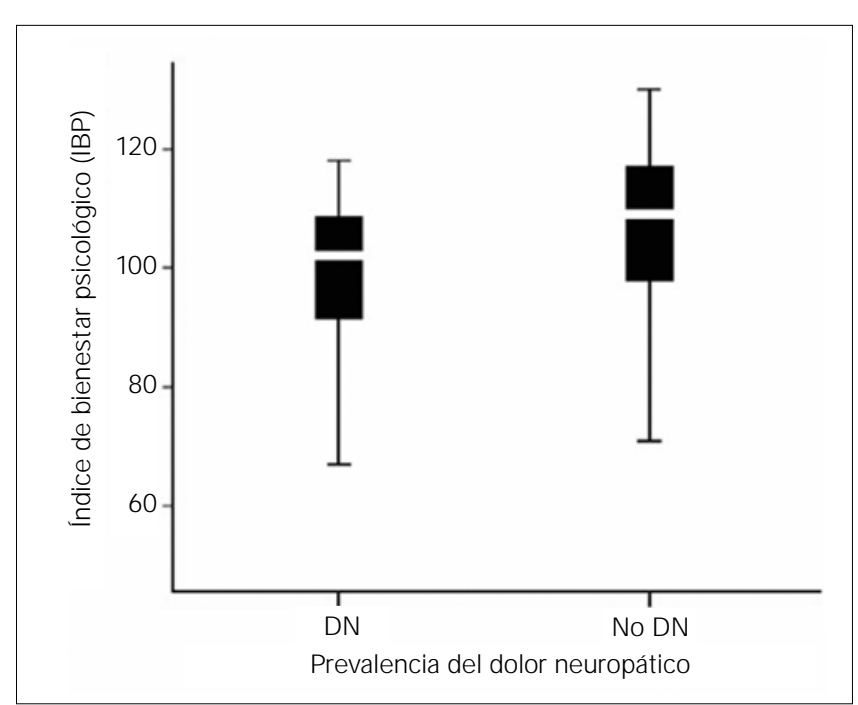

Figura 4. Prevalencia del dolor neuropático en relación con la media de puntuación del IBP.

Tabla III. Puntuaciones del índice de bienestar psicológico (IBP) y de sus subescalas.

\begin{tabular}{|c|c|c|c|c|c|c|}
\hline & & $n$ & Media & DE & $F$ & $p$ \\
\hline \multirow[t]{3}{*}{ IBP } & DN & 87 & 98,18 & 15,02 & \multirow{3}{*}{14.789} & \multirow{3}{*}{$0,000^{b}$} \\
\hline & Control & 110 & 106,28 & 14,40 & & \\
\hline & Total & 197 & 102,71 & 15,18 & & \\
\hline \multirow[t]{3}{*}{ Salud general } & DN & 84 & 11,36 & 3,06 & \multirow{3}{*}{68.436} & \multirow{3}{*}{$0,000^{b}$} \\
\hline & Control & 110 & 14,87 & 2,83 & & \\
\hline & Total & 194 & 13,35 & 3,41 & & \\
\hline \multirow[t]{3}{*}{ Bienestar } & DN & 84 & 14,58 & 3,48 & \multirow{3}{*}{13.371} & \multirow{3}{*}{$0,000^{b}$} \\
\hline & Control & 110 & 16,45 & 3,54 & & \\
\hline & Total & 194 & 15,64 & 3,62 & & \\
\hline \multirow[t]{3}{*}{ Depresión } & DN & 84 & 15,46 & 2,92 & \multirow{3}{*}{6.661} & \multirow{3}{*}{$0,011^{a}$} \\
\hline & Control & 110 & 16,36 & 1,92 & & \\
\hline & Total & 194 & 15,97 & 2,44 & & \\
\hline \multirow[t]{3}{*}{ Vitalidad } & $\mathrm{DN}$ & 84 & 17,13 & 2,89 & \multirow{3}{*}{5.385} & \multirow{3}{*}{$0,021^{a}$} \\
\hline & Control & 110 & 18,13 & 3,01 & & \\
\hline & Total & 194 & 17,70 & 2,99 & & \\
\hline \multirow[t]{3}{*}{ Ansiedad } & DN & 84 & 24,05 & 4,53 & \multirow{3}{*}{1672} & \multirow{3}{*}{0,198} \\
\hline & Control & 110 & 24,89 & 4,48 & & \\
\hline & Total & 194 & 24,53 & 4,51 & & \\
\hline \multirow[t]{3}{*}{ Autocontrol } & DN & 84 & 15,15 & 2,82 & \multirow{3}{*}{1176} & \multirow{3}{*}{0,279} \\
\hline & Control & 110 & 15,58 & 2,64 & & \\
\hline & Total & 194 & 15,40 & 2,72 & & \\
\hline
\end{tabular}

${ }^{\mathrm{a}} p<0,05 ;{ }^{\mathrm{b}} p<0,01 \mathrm{DE}$ : desviación estándar; DN: dolor neuropático.

sencia de DN y la percepción de bienestar psicológico: los pacientes con DN mostraban un nivel de sufrimiento mayor que los sujetos sin dolor. Con relación a los diferentes tipos de DN (a nivel o por debajo), la variable con valor predictivo en la clasificación de DN fue el inicio temprano del dolor en el DN a nivel, y la lesión incompleta en el DN por debajo de la lesión.

Nuestros resultados de prevalencia del DN son muy similares a los obtenidos en estudios previos $[3,21,35]$ en otras poblaciones, lo cual apoya la fiabilidad y la validez del sistema de clasificación empleado en nuestro estudio. En cuanto a la distribución del dolor en las categorías de DN a nivel y por debajo, nuestros datos coinciden con otros estudios que han empleado la misma clasificación, como los de Werhagen et al [21] (a nivel, 12\%; por debajo, 28\%) y Barrett et al [35] (a nivel, 11,4\%; por debajo, 24,3\%); el grupo con dolor por debajo es el más numeroso. En contraste, en el estudio longitudinal de Siddall et al [3], el dolor a nivel se da con mayor frecuencia. Posibles razones sobre estas diferencias podrían ser el diseño del estudio así como las características de la muestra. En el estudio de Siddall et al [3] la media de edad de la muestra es de 40 años (eran más jóvenes que en nuestra muestra); además, la etiología de la lesión era sólo traumática y el tiempo transcurrido tras la lesión era menor que en nuestro estudio.

Entre las variables demográficas, uno de los hallazgos destacables es la relación entre la aparición del dolor con la edad en el momento de la instauración de la lesión y la edad en el momento de la entrevista. Los pacientes que sufren la lesión medular a edades más tempranas tienen un riesgo menor de desarrollar dolor crónico. Este hallazgo coincide con lo publicado por varios autores [20,21,23,24,36]. Posibles explicaciones a este hecho pueden incluir el declive con la edad de la plasticidad cerebral en el sistema nervioso; por consiguiente, disminuye la capacidad de modulación en el delicado equilibrio de la transmisión de las señales nocioceptivas hacia el sistema nervioso central [21]. Estudios recientes de neuroquímica también demuestran que los niveles espinales de serotonina y norepinefrina, neurotransmisores implicados en el sistema inhibitorio descendente del dolor, decrecen en función de la edad [37].

En la mayor parte de nuestros pacientes, el dolor aparece durante el primer año tras la LM. No obstante, existe un porcentaje pequeño de personas $(8,5 \%$, de las cuales el $80 \%$ tiene dolor por debajo de la lesión) en las que el DN aparece a partir de los dos años después de la LM; nuestros resultados coinciden con los de otros estudios [2,35]. Nuestros datos indican que la larga duración de la discapacidad no está asociada con una frecuencia mayor del dolor. Este dato coincide con los hallados por otros investigadores $[6,26,38]$. Es necesario tener en cuenta que en estos últimos estudios se analiza de forma global el dolor; se mezclan el DN causado por la misma lesión en la médula y el dolor musculoesquelético que se desarrolla en el tiempo, asociado al esfuerzo requerido por la movilidad. El poder analizar por separado los diferentes tipos de dolor permite comprobar que éstos tienen diferentes momentos de inicio tal y como refleja el estudio de Siddall et al [3]. El dolor a nivel de la lesión tiene un inicio precoz, pero el dolor por debajo de la lesión tiene un inicio más tardío; los datos coinciden con trabajos anteriores [3]. Este inicio más tardío, tal y como afirman Siddall et al [3], puede estar reflejando diferencias en los mecanismos subyacentes responsables de la generación del dolor.

En nuestro estudio, el DN se comunica como grave en un porcentaje elevado de personas; estos datos son similares a los encontrados por otros autores [2,3]. La gravedad del dolor no se ha relacionado con ninguna de las dos categorías de DN. Tampoco se ha detectado una relación significativa entre la gravedad 
del dolor y las variables médicas; estos datos coinciden con los de estudios anteriores [8,20,27].

Otro aspecto interesante que hemos observado es una frecuencia mayor en la presentación del DN por debajo de la lesión en las lesiones medulares incompletas. Esta relación resulta controvertida, de modo que mientras algunos estudios la apoyan $[20,22,24,39]$ otros indican el sentido contrario [3,5,21]. En la explicación fisiopatológica del DN se ha postulado una alteración de la representación de la imagen corporal, generada por un cambio en la integración sensorial y motora a distintos niveles. Werhagen et al [21] sugieren que, en las lesiones incompletas, la alteración de la integración podría resultar más compleja como consecuencia de un grado distinto de afectación a los distintos niveles, y justifican una mayor incidencia de DN por debajo de la lesión.

Nuestro estudio muestra que el dolor impacta negativamente en la percepción de bienestar psicológico de los individuos; estas personas tienen una percepción de la salud, la vitalidad y el bienestar más baja que el grupo control, así como un humor más depresivo. Estos hallazgos se asemejan a la bibliografía ya existente $[3,7,35,39,40]$. Pese a que es difícil establecer relaciones de causa-efecto entre el dolor y el malestar emocional, estudios como el de Cairns et al [41] indican que reducir la intensidad del dolor tiene un efecto muy positivo sobre la depresión más que la reducción de la depresión sobre el dolor. Fishbain et al [42], en su revisión sistemática sobre el dolor crónico en general, concluyeron que un dolor persistente es más probable que conduzca a una depresión que viceversa y que los pacientes con un dolor más frecuente e intenso tienen un riesgo mayor de depresión grave. Chapman et al [43] sugieren que el dolor crónico es un potente estresante que promueve una respuesta de estrés extensa y destructiva que implica una desregulación neuroendo- crina, fatiga, disforia, mialgia y que afecta al estado de ánimo y a la capacidad funcional de los pacientes. Estos datos apoyan la necesidad de evaluar los aspectos emocionales de las personas que refieren dolor y, en caso necesario, abordarlos como parte del tratamiento del control del dolor.

Las estrategias de evaluación y tratamiento para el DN crónico deberían basarse en la fenomenología del dolor, en los mecanismos fisiopatológicos de éste, así como en los aspectos psicológicos y los aspectos biológicos en relación con la naturaleza plástica del sistema nervioso.

En conclusión, en la población de estudio hemos hallado que: - La prevalencia del DN tras una lesión medular es del 35\%. - La edad avanzada en el momento de la lesión (> 50 años) es un factor de riesgo en el desarrollo de este tipo de dolor.

- Las lesiones incompletas tienen una probabilidad mayor de desarrollar DN por debajo de la lesión.

- El DN a nivel de la lesión tiene un inicio más temprano tras la instauración de la lesión medular.

- El dolor impacta de forma negativa en la percepción global de bienestar psicológico.

Estos resultados apoyan la idea de que el sistema de clasificación del dolor empleado en nuestro estudio puede reflejar diferentes mecanismos subyacentes en las categorías de DN. Pensamos que es un buen punto de partida y anima a proseguir en el estudio de los mecanismos y los factores que intervienen en la experiencia del dolor.

La prevalencia elevada, el inicio temprano y la intensidad de este tipo de dolor así como su tendencia a la cronificación determinan la importancia de la valoración y el tratamiento en los estadios más iniciales.

\section{BIBLIOGRAFÍA}

1. Siddall PJ, Loeser JD. Pain following spinal cord injury. Spinal Cord 2001; 39: 63-73.

2. Störmer S, Gerner HJ, Gruninger W, Metzmacher K, Follinger S, Wien$\mathrm{ke} \mathrm{C}$, et al. Chronic pain/dysaesthesiae in spinal cord injury patients: results of a multicentre study. Spinal Cord 1997; 35: 446-55.

3. Siddall PJ, McClelland JM, Rutkowski SB, Cousins MJ. A longitudinal study of the prevalence and characteristics of pain in the first 5 years following spinal cord injury. Pain 2003; 103: 249-57.

4. Turner JA, Cardenas DD, Warms CA, McClellan CB. Chronic pain associated with spinal cord injuries: a community survey. Arch Phys Med Rehabil 2001; 82: 501-9.

5. Ravenscroft A, Ahmed YS, Burnside IG. Chronic pain after SCI. A patient survey. Spinal Cord 2000; 38: 611-4.

6. Rintala DH, Loubser PG, Castro J, Hart KA, Fuhrer MJ. Chronic pain in a community-based sample of men with spinal cord injury: prevalence, severity, and relationship with impairment, disability, handicap, and subjective well-being. Arch Phys Med Rehabil 1998; 79: 604-14.

7. Widerström-Noga EG, Felipe-Cuervo E, Broton JG, Duncan RC, Yezierski RP. Perceived difficulty in dealing with consequences of spinal cord injury. Arch Phys Med Rehabil 1999; 80: 580-6.

8. Richards JS, Meredith RL, Nepomuceno C, Fine PR, Bennett G. Psycho-social aspects of chronic pain in spinal cord injury. Pain 1980; 8: 355-66.

9. King C, Kennedy P. Coping effectiveness training for people with spinal cord injury: preliminary results of a controlled trial. Br J Clin Psychol 1999; 38: 5-14.

10. Scivoletto G, Petrelli A, Di Lucente L, Castellano VI. Psychological investigation of spinal cord injury patients. Spinal Cord 1997; 35: 516-20.

11. Siddall PJ, Yezierski RP, Loeser JD. Pain following spinal cord injury: clinical features, prevalence, and taxonomy. IASP Newsletter 2000; 3: 3-7.

12. Siddall PJ, Taylor DA, Cousins MJ. Classification of pain following spinal cord injury. Spinal Cord 1997; 35: 69-75.

13. Yezierski RP. Pain following spinal cord injury: pathophysiology and central mechanisms. Prog Brain Res 2000; 129: 429-49.
14. Tator CH, Fejlings MG. Review of the secondary injury theory of acute spinal cord trauma with emphasis on vascular mechanisms. J Neurosurg 1991; 75: 100-5.

15. Bethea JR, Castro M, Keane RW, Lee TT, Dietrich WD, Yezierski RP. Traumatic spinal cord injury induces nuclear factor-kappa B activation. J Neurosci 1998; 18: 3251-60.

16. Vierck Jr. CJ, Siddall P, Yezierski RP. Pain following spinal cord injury: animal models and mechanistic studies. Pain 2000; 89: 1-5.

17. Bowsher D. Central pain: clinical and physiological characteristics. J Neurol Neurosurg Psychiatry 1996; 61: 62-9.

18. Yezierski RP. Pain following spinal cord injury: the clinical problem and experimental studies. Pain 1996; 68: 185-94.

19. Finnerup NB, Jensen TS. Spinal cord injury pain-mechanisms and treatment. Eur J Neurol 2004; 11: 73-82.

20. Siddall PJ, Taylor DA, McClelland JM, Rutkowski SB, Cousins MJ. Pain report and the relationship of pain to physical factors in the first 6 months following spinal cord injury. Pain 1999; 81: 187-97.

21. Werhagen L, Budh CN, Hultling C, Molander C. Neuropathic pain after traumatic spinal cord injury-relations to gender, spinal level, completeness, and age at the time of injury. Spinal Cord 2004; 42: 665-73.

22. Finnerup NB, Johannesen IL, Sindrup SH, Bach FW, Jensen TS. Pain and dysesthesia in patients with spinal cord injury: a postal survey. Spinal Cord 2001; 39: 256-62.

23. Rintala DH, Hart KA, Priebe MM. Predicting consistency of pain over a 10 -year period in persons with spinal cord injury. J Rehabil Res Dev 2004; 41: 75-88.

24. Budh CN, Lund I, Hultling C, Levi R, Werhagen L, Ertzgaard P, et al. Gender related differences in pain in spinal cord injured individuals. Spinal Cord 2003; 41: 122-8.

25. Cardenas DD, Bryce TN, Shem K, Richards JS, Elhefni H. Gender and minority differences in the pain experience of people with spinal cord injury. Arch Phys Med Rehabil 2004; 85: 1774-81.

26. Demirel G, Yllmaz H, Gençosmanoglu B, Kesiktas N. Pain following spinal cord injury. Spinal Cord 1998; 36: 25-8. 
27. Summers JD, Rapoff MA, Varghese G, Porter K, Palmer RE. Psychosocial factors in chronic spinal cord injury pain. Pain 1991; 47: 183-9.

28. ASIA. International standards for neurological and functional classification of spinal cord injury. Chicago: American Spinal Injury Association; 1996.

29. Maynard FM, Bracken MB, Creasey G, Ditunno JF, Donovan WH, Ducker TB, et al. International standards for neurological and functional classification of spinal cord injury. Spinal Cord 1997; 35: 266-74.

30. Widerström-Noga EG, Cruz-Almeida Y, Krassioukov A. Is there a relationship between chronic pain and autonomic dysreflexia in persons with cervical spinal cord injury? J Neurotrauma 2004; 21: 195-204.

31. Widerström-Noga EG, Felipe-Cuervo E, Yezierski RP. Relationships among clinical characteristics of chronic pain after spinal cord injury. Arch Phys Med Rehabil 2001; 82: 1191-7.

32. Williams AC, Davies HT, Chadury Y. Simple pain rating scales hide complex idiosyncratic meanings. Pain 2000; 85: 457-63.

33. Dupuy HJ. The Psychological General Well-Being (PGWB) Index. In Wenger NK, Mattson ME, Furberg CD, Elinson J, eds. Assessment of quality of life in clinical trials of cardiovascular therapies. New York: Le Jacq Publishing; 1984. p. 170-83.

34. Badía X, Gutiérrez F, Wiklund I, Alonso J. Validity and reliability of Spanish version of the General Well Being Index. Qual Life Res 1996; 5: 101-8.

35. Barret H, McClelland MJ, Rutkowski SB, Siddall PJ. Pain characteris- tics in patients admitted to hospital with complications after spinal cord injury. Arch Phys Med Rehabil 2003; 84: 789-95.

36. Putzke JD, Richards JS, Hicken BL, De Vivo MJ. Interference due to pain following spinal cord injury: important predictors and impact on quality of life. Pain 2002; 100: 231-42.

37. Ko ML, King MA, Gordon TL, Crisp T. The effects of aging on spinal neurochemistry in the rat. Brain Res Bull 1996; 42: 95-8.

38. Widerström-Noga EG, Duncan R, Felipe-Cuervo E, Turk DC. Assessment of the impact of pain and impairments associated with spinal cord injuries. Arch Phys Med Rehabil 2002; 83: 395-404.

39. Kennedy P, Frankel H, Gardner B, Nuseibeh I. Factors associated with acute and chronic pain following traumatic spinal cord injuries. Spinal Cord 1997; 35: 814-7.

40. Wagner AG, Stenehjem AE, Stanghelle JK. Pain and life quality within 2 years of spinal cord injury. Paraplegia 1995; 33: 555-9.

41. Cairns MD, Adkins RH, Scott MD. Pain and depression in acute traumatic spinal cord injury: origins of chronic problematic pain? Arch Phys Med Rehabil 1996; 77: 329-35.

42. Fishbain DA, Cutler R, Rosomoff HL, Rosomoff RS. Chronic pain-associated depression: antecedents or consequence of chronic pain? A review. Clin J Pain 1997; 13: 116-37.

43. Chapman RC, Gavrin J. Suffering: the contributions of persistent pain. Lancet 1999; 353: 2233-7.

\section{CHARACTERISTICS OF CHRONIC NEUROPATHIC PAIN AND THEIR RELATIONSHIP WITH PSYCHOLOGICAL WELL-BEING IN SPINAL CORD INJURY PATIENTS}

Summary. Aims. To determine the prevalence of neuropathic pain (NP) in a sample of patients with spinal cord injury (SCI), to examine the relation between clinical, demographic and psychological characteristics with the prevalence of NP after SCI and the different types of NP, and to study predictive factors. Patients and methods. Transversal observational study of 260 patients who attended their annual revision. Main outcome measures included presencelabsence of NP, psychological wellbeing index, detailed pain history and 0-10 numerical rating scale of average pain intensity. Results. 93 patients (36\%) with SCI had NP. Of the patients with NP, 35\% showed pain at level, while 65\% showed NP below the level of injury. The only variable with a predictive value on the prevalence of NP was older age at the time of SCI. Statistically significant differences were observed between the prevalence of NP and the perception of psychological well-being; patients with NP were the ones with more psychological distress. As for the different types of NP, the variables with predictive value on the classification of $N P$ were early onset of pain at level, and incompleteness of the lesion in the below level NP. Conclusion. The results of this study show that NP appears frequently after SCI being more prevalent in patients who suffered the injury at more advanced ages. NP has a negative impact on the perception of psychological well-being. [REV NEUROL 2007; 44: 3-9]

Key words. Chronic neuropathic pain. Pain at level. Pain below level. Prevalence. Psychological well-being. Spinal cord injuries. 